\title{
Prevalence of Urinary Tract Infection in Patient with Indwelling Catheter in Chittagong Medical College Hospital
}

\author{
Raushan Akter ${ }^{1 *}$ \\ Mahtab Uddin Hassan ${ }^{2}$ \\ Rajat Sankar Roy Biswas ${ }^{1}$
}

${ }^{1}$ Department of Medicine

Chattagram Maa Shishu-O-General Hospital Chittagong, Bangladesh.

${ }^{2}$ Department of Medicine

Marine City Medical College Hospital Chittagong, Bangladesh.

\section{*Correspondence to:}

\section{Dr. Raushan Akter}

Resident Physician

Department of Medicine

Chattagram Maa Shishu-O-General Hospital

Chittagong, Bangladesh.

Mobile : +88 01556541538,01683679317

Email : rushanakter@yahoo.com

www.banglajol.info/index.php/CMOSHMCJ

\begin{abstract}
Background: Hospital acquired urinary tract infection among the hospitalized catheterized patient due to different indications are a common morbility. The objective of study to see the prevalence hospital acquired urinary tract infection among the patients of indwelling catheter admitted in the department Medicine of Chittagong Medical College Hospital. Methods: It was a hospital based obsevational study conducted on purposively selected (Non-probability) hospitalized patients who got indwelling catheter had admitted into the Medicine ward, Chittagong Medical College Hospital from $1^{\text {st }}$ January 2013 to $30^{\text {th }}$ June 2013. Total 50 patients were selected. Urine was analyzed for growth of organism and sensitivity was also done in case where growth was positive. Data was analyzed by statistical method with the software SPSS-20. Results: Results showed that 15 (30\%) developed bacteriuria or urinary tract infection with catheter. Development of bacteriuria was not affected by sex, age. People who introduce the catheter has some influence with the occurrence of UTI. E.coli, Klebsella, Proteas and Pseudomonas aeroginosa were the most common bacteria isolated. Imepenam was found the most sensitive antibiotic. Conclusion: Catheter play an important risk for occurrence of UTI. So it should be avoided if possible.
\end{abstract}

Key words: Urinary tract infection; Indwelling urinary catheters; Bacteriuria; Pseudomonas aeroginosa; E.col.

\section{INTRODUCTION}

Nosocomial infections are significant source of morbidity and mortality. It also means extra expenses once it sets in because of prolonged hospital stay and additional hospital needs ${ }^{1}$. Because of theses, activities toward prevention and control of hospital acquired infections have been made since the 1950's.

In the United States, nosocomial infections occur in about $5 \%$ of patients admitted in acute care hospitals. In a surveillance study done by the infection control committee of the Philippine General Hospital (PGH) in 1989, the prevalence was $16.3 \%^{2}$.

Nosocomial urinary tract infection remain a significant contributor to the overall prevalence or incidence of nosocomial infection. Urethral catheter is a major predisposing factor, caused by variety of pathogens, including E.coli, Klebsella, Proteas and Pseudomonas aeroginosa, enterobecter,serratia and candida.Many of theses microorganisms are part of the patient's endogenous bowel flora,but they can also be acquired by cross transmission from other patients or hospital personnel or by exposure to contaminated solutions or non sterile equipment. Situations like immunosuppression, steroid intake, or antibiotic intake would change the usual patterns of pathogens in catheter associated bacteriuria ${ }^{3,4}$. 
In between 20 and 30 percent of catheterised patients develop bacteriuria, of whom 2-6 percent develop symptoms of urinary tract infection. The risk of acquiring bacteriuria is approximately $5 \%$ for each day of catherisation ${ }^{5}$.

Indwelling urinary catheters pose a risk for many infective complications such as perinephric, vesical and urethral abscesses as well as epididymitis,prostatitis and orchitis. The overall incidence of theses complications is unknown, although 20 to 30 percents with asymptomatic catheter induced UTIs may develop local or systematic symptoms ${ }^{6}$. Of patients with a UTI, 1-4 \% develops becterimia and, of theses, 13-30\% die. Duration of catheterisation is strongly associated with risk of infection $\mathrm{i}$ e. the longer the catheter is in place, the higher the incidence of $\mathrm{UTI}^{3,4}$.

A study was done in Chittagong Medical College Hospital to see the prevalence of HAI according to WHO guideline. The point prevalence of HAI was found $16.9 \%{ }^{7}$.

The type of HAI found in $\mathrm{CMCH}$ in this prevalence survey were UTI $=25.8 \%$,surgical site infection $65.6 \%$, pneumonia $9.7 \%$, blood stream infection $15 \%$, gastroenteritis $4.3 \%{ }^{7}$.

The predominant organism identified were klebsella $(32.7 \%)$ Coliforms (23.6\%) Pseudomonas (29.1\%) E.coli (20\%) S. aurius (3.6\%) Enterococci $(1.8 \%)$ and proteus $(1.8 \%)^{7}$. Prevention of nosocomial UTI and its complications would therefore reduce the rate of morbidity and mortality and expenses secondary to the infection. This study is aimed to determine the incidence of bacteriuria, its onset after insertion, and the predisposing factors following insertion of indwelling catheter.

\section{MATERIALS AND METHODS}

This observational study was conducted at department of Medicine in Chittagong Medical College Hospital (CMCH) Chittagong. Duration of study was 6 (Six) months. Patients admitted in the Department of medicine and who were given Foley Catheter during that period were included in this study. A total of 50 patients were included. Data was collected by interview, physical and laboratory examinations using a structured questionnaire. Urine sample was collected in a clean dry container and for microscopic examination and culture. $\mathrm{RBC}$, pus cell was determined and urine albumin also detected. Culture was done in Mackconkey's agar and blood agar. Coloney count were calculated if growth was present .Five ml of peripheral blood was collected for examnation of total count and differential count of WBC.Data was processed \& analyzed by SPSS-20 version. Different statistical method will be applied for data analysis.

\section{RESULTS}

In our study total 50 patients were included. 33(60\%) were male \& $17(34 \%)$ were female. Male to female ratio was $1.9: 1$. Most of the patients age was 51 and above. $78 \%$ patients came from rural area \& $22 \%$ were from urban area. Among the patients, $70 \%$ had the indications of neurogical bladder dysfunction. The common underlying illness were stroke \& OPC poisoning. Frequency of UTI was $30 \%$ among 50 catheterized patients where male were found more diseased than female (18 $\%$ vs $12 \%$ ). It was observed that prevalence was found among those patients who were catheterized by non trained person (50\%). Infectious agent was identified in $30 \%$ by urine culture. Pathogens most commonly demonstrated was E. coli 9(60\%) followed by Klebsiella 3(20\%), Proteus $2(13.3 \%) \&$ pseudomonas $1(6.6 \%)$. E.coli was sensitive to Amoxicillin, Ceftriaxone, Imipenem, Meropenem, Vancomycin and resistant to Ciprofloxacin, Amoxyclav and gentamycin. Pseudomonas sp. was sensitive to ciprofloxacin, gentamycin, ceftazidime, meropenem and vancomycin. This organism was resistance to amoxicillin, cephalexin and azithromycin. Klebsiella pneumonae were sensitive to ceftraixone, ciprofloxacin, gentamycin, imipenem \& meropenem. This organism was resistance only to amoxicillin. S. pyogen were sensitive to amoxicillin, ceftriaxone, cephalexin, imipenem, meropenem and vancomycin

Table 1 : Aga distribution of the patients $(n=50)$.

$\begin{array}{lcc}\text { Age group (Years) } & \text { Number } & \text { Percentage } \\ <40 & 5 & 10 \% \\ 41-50 & 6 & 12 \% \\ 51-60 & 14 & 28 \% \\ 61-70 & 21 & 42 \% \\ >70 & 4 & 8 \%\end{array}$

Table 2 : Gender distribution of the patients $(n=50)$.

\begin{tabular}{lcc} 
Sex & Number & Percentage \\
Male & 33 & $66 \%$ \\
Female & 17 & $34 \%$ \\
\hline
\end{tabular}

Table 3 : Distribution of patients according to indications of catheterization $(\mathrm{n}=50)$.

\begin{tabular}{lcc} 
Indications of & Number & Percentage \\
catheterization & n & \% \\
Incontinence & 4 & 8.0 \\
Retention & 6 & 12.0 \\
Output monitoring & 3 & 6.0 \\
Neurological bladder & & \\
dysfunction & 35 & 70.0 \\
Urinary obstruction & 2 & 4.0 \\
\hline
\end{tabular}

Table 4 : Distribution of common underlying illness $(n=50)$.

\begin{tabular}{lcc} 
Diagnosis & Number & Percentage \\
Pneumonia & 4 & $8 \%$ \\
Hypertension & 3 & $6 \%$ \\
Stroke & 16 & $32 \%$ \\
PTB & 5 & $10 \%$ \\
OPC poisoning & 11 & $22 \%$ \\
DM & 4 & $8 \%$ \\
COPD & 7 & $14 \%$ \\
\hline
\end{tabular}




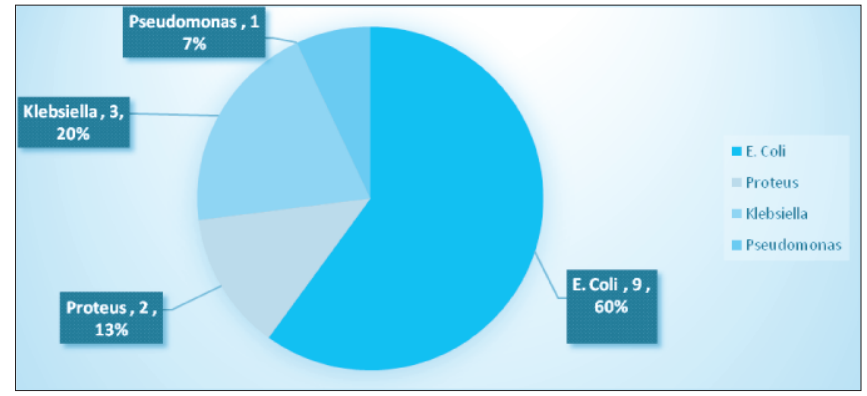

Figure 1: Pie chart showing different types of organism that were identified by culture.

Table 5 : Table showing antibiogram of E coil in UTI.

\begin{tabular}{lccc} 
Drugs & Sensitive & Moderately Sensitive Resistant \\
Amoxicillin & 0 & 1 & 8 \\
Azythromycin & 1 & 1 & 7 \\
Nitrofurantoin & 5 & 1 & 3 \\
Netilmycin & 5 & 1 & 3 \\
Gentamycin & 6 & 0 & 3 \\
Amikacin & 4 & 0 & 5 \\
Meropenem & 9 & 0 & 0 \\
Ciprofloxacin & 2 & 1 & 6 \\
Cefotaxime & 3 & 1 & 5 \\
Ceftazidime & 4 & 1 & 4 \\
Ceftriaxone & 3 & 0 & 6 \\
Cotrimoxazole & 5 & 1 & 3 \\
Cefixime & 5 & 3 & 1 \\
\hline
\end{tabular}

Table 5 showing the pattern of sensitivity of E coil where it was found that E. was sensitive Meropenem and resistant to Amoxycillin, Ciprofluxacine and Gentamycin. Some drugs showed moderate sensitivity against this bacteria.

Table 6 : Table showing antibiogram of Klebsiella in UTI $(n=3)$.

$\begin{array}{lccc}\text { Drugs } & \text { Sensitive } & \text { Moderately Sensitive } & \text { Resistant } \\ \text { Amoxicillin } & 0 & 0 & 3 \\ \text { Azythromycin } & 1 & 0 & 2 \\ \text { Nitrofurantoin } & 2 & 0 & 1 \\ \text { Netilmycin } & 2 & 1 & 0 \\ \text { Gentamycin } & 1 & 0 & 2 \\ \text { Amikacin } & 2 & 1 & 0 \\ \text { Meropenem } & 3 & 0 & 0 \\ \text { Ciprofloxacin } & 0 & 1 & 2 \\ \text { Cefotaxime } & 1 & 0 & 2 \\ \text { Ceftazidime } & 1 & 0 & 2 \\ \text { Ceftriaxone } & 0 & 0 & 3 \\ \text { Cotrimoxazole } & 0 & 1 & 2 \\ \text { Cefixime } & 1 & 2 & 0\end{array}$

Table 6 showing pattern of sensitivity of Klebsiella where the second common bacteria Klebsiella was found sensitive to Meropenem in full and other antibiotics were moderate to full sensitive. Amoxycillin, Ciprofluxacine and Ceftriaxone was found insensitive.
Table 7 : Table showing overall sensitivity pattern of antibiotics in UTI $(n=15)$.

\begin{tabular}{lccc} 
Drugs & Sensitive & Moderately Sensitive & Resistant \\
Amoxicillin & 0 & 0 & 15 \\
Azythromycin & 3 & 1 & 11 \\
Nitrofurantoin & 9 & 1 & 5 \\
Netilmycin & 9 & 1 & 5 \\
Gentamycin & 4 & 0 & 11 \\
Amikacin & 7 & 1 & 7 \\
Meropenem & 15 & 0 & 0 \\
Ciprofloxacin & 2 & 1 & 12 \\
Cefotaxime & 3 & 0 & 12 \\
Ceftazidime & 6 & 1 & 8 \\
Ceftriaxone & 4 & 0 & 11 \\
Cotrimoxazole & 7 & 2 & 6 \\
Cefixime & 5 & 9 & 1 \\
\hline
\end{tabular}

Table 7 showing overall sensitivity pattern antibiotics in UTI of catheterized patients where among 13 antibiotics Meropenem was found highly sensitive to all bacteria. On the contrary Amoxicillin was found resistant to all of them. Nitrofurantoin was found highly sensitive to resistant pattern.

\section{DISCUSSION}

Present study wass done in the Medicine Department of $\mathrm{CMCH}$ among the 50 patients who were given indwelling foley catheter to evaluate the presence of Urinary Tract Infection (UTI) among them.

Regarding the analysis of demographic variables age of the most patients were above 51 and male patients were $33(66 \%)$ and female patient were 17 (34\%). As indwelling catheter mostly needed for the elderly, the present age distribution can be explained in that way. Female has less excess to hospital care due to financial and social factors, so number of female patients may less than the male patients.

Underlying disease which in the present study was stroke and OPC poisoning which was $32 \%$ and $22 \%$ respectively. Disease profile of $\mathrm{CMCH} 2012$ and an ongoing study correlates these findings ${ }^{7}$.

Prevalence of UTI in the present study was 30\%. Nosocomial UTI occasionally results from direct introduction of urethral microorganisms at the time of catheterization. Microorganisms can migrate to the bladder along the outside of the catheter in the peri-urethral mucosal sheath (Between the catheter and the urethral mucosa), the trans- urethral route, or along the internal lumen of the catheter after the collection bag or catheter drainage tube junction has been contaminated (intraluminal). A study done in $\mathrm{CMCH}$ found the prevalence of UTI as a hospital acquired infection was $25.8 \%$ which also correlate the present findings ${ }^{7}$. 
Catheter associated UTI were caused by variety of pathogens, including Escherichia coli which was most common (60\%), next to it were Klebsiella, Proteus and Pseudomonas.Many of these microorganisms are part of the patient's endogenous bowel flora, but they can also be acquired by cross- transmission from other patients or hospital personnel or by exposure to contaminated solutions or non sterile equipment ${ }^{1}$.

Study done in $\mathrm{CMCH}$ regarding pathogen causing hospital acquired infection found that E. coli was $20 \%$ and Klebsiella was $32.7 \%$. That study was done on overall hospital acquired infection where as the present study was on the patients with indwelling catheter where catheter played an important extra risk factor for them. Regarding antibiotic sensitivity most of the organism were resistant to common antibiotics. Imipenem was the only antibiotic which was found sensitive to $100 \%$ of all organism. This finding was also consistent with the previous study ${ }^{7}$.

\section{CONCLUSION}

UTI is a common nosocomial infection among the catheterized hospitalized patients. Proper care should be taken during introduction and drainage of the catheter.

\section{DISCLOSURE}

All the authors declared no competing interest.

\section{REFERENCES}

1. CDC. Guidelines for Prevention of catheter. Related UTI. In: Guidelines for Prevention \& Control of Nosocomial Infection Centre for Disease Control, Atlantia. 1982.

2. ICC Bulletin,PGH Infection Control Comittee. Nosocomial Infection Prevalence Study in PGH for. 1989;1990;(1).

3. Wong ES, Hooton TM. Guideline for prevention of catheter-associated urinary tract infections. Am Jour inf Cont.1983; 11(1) : 28-36.

4. Saint S. Clinical and economic consequences of nosocomial catheter related bacteriuria. Am J Infect Control. 2000; 28:68-75.

5. Erikson NH . Poulsen PN. Urinary tract infection : Etiology, Diagnosis and treatment with effective antibiotics.Nord Medicine. 1989; 104(2): 35-38.

6. Asher EF,Oliver BG, Fry DE, Urinary tract infection in surgical patients.Am J Surg. 1988 ;54(7):466-469.

7. Yusuf O F Hassan M U et al. Draft reports on a survey of hospital acquired infection in Chittagong Medical College Hospital 2010. 